\title{
Multi-Feature Based Emotion Recognition for Video Clips
}

\author{
Chuanhe Liu \\ Situ AI Labs Beijing \\ Beijing, 100089, China \\ liuchuanhe@situdata.com
}

\author{
Tianhao Tang \\ Situ AI Labs Beijing \\ Beijing, 100089, China \\ tangtianhao@situdata.com \\ Minghao Wang \\ Situ AI Labs Beijing \\ Beijing, 100089, China \\ wangmh@situdata.com
}

\author{
Kui Lv* \\ Situ AI Labs Beijing \\ Beijing, 100089, China \\ lvkui@situdata.com
}

\begin{abstract}
In this paper, we present our latest progress in Emotion Recognition techniques, which combines acoustic features and facial features in both non-temporal and temporal mode. This paper presents the details of our techniques used in the Audio-Video Emotion Recognition subtask in the 2018 Emotion Recognition in the Wild (EmotiW) Challenge. After the multimodal results fusion, our final accuracy in Acted Facial Expression in Wild (AFEW) test dataset achieves $61.87 \%$, which is $1.53 \%$ higher than the best results last year. Such improvements prove the effectiveness of our methods.
\end{abstract}

\section{CCS CONCEPTS}

- Computing methodologies $\rightarrow$ Appearance and texture representations; Neural networks;

\section{KEYWORDS}

Emotion Recognition; EmotiW 2018; Deep Learning; SoundNet; Inception Net; DenseNet; LSTM; 3D Face Landmark

\section{ACM Reference format:}

Chuanhe Liu, Tianhao Tang, Kui Lv and Minghao Wang. 2018. MultiFeature Based Emotion Recognition for Video Clips. In Proceedings of 20th ACM International Conference on Multimodal Interaction (ICMI'18), October 16-20, 2018, Boulder, CO, USA. ACM, NY, NY, USA, 4 pages.

https://doi.org/10.1145/3242969.3264989

\section{INTRODUCTION}

There is a long history in automatic emotion recognition. It has been proved to be one of the most challenging tasks in computer

*Kui Lv is an intern at the Beijing Situ Data Technology Service Co.Ltd of Beijing University of Chemical Technology when doing the works.

Permission to make digital or hard copies of all or part of this work for personal or classroom use is granted without fee provided that copies are not made or distributed for profit or commercial advantage and that copies bear this notice and the full citation on the first page. Copyrights for components of this work owned by others than ACM must be honored. Abstracting with credit is permitted. To copy otherwise, or republish, to post on servers or to redistribute to lists, requires prior specific permission and/or a fee. Request permissions from Permissions@acm.org.

ICMI '18, October 16-20, 2018, Boulder, CO, USA

(C) 2018 Association for Computing Machinery.

ACM ISBN 978-1-4503-5692-3/18/10...\$15.00

https://doi.org/10.1145/3242969.3264989 vision. For years, researchers have been working with manually designed features to identify people's emotions based on facial expression. Former researchers have achieved some remarkable progress while such technique still has its limitations. In recent years, with the development of deep learning, facial features extracted by the deep neural network has been proved to be a huge step forward in emotion recognition.

The EmotiW [1] challenges have been held successfully since 2013. The Audio Video sub-challenge is to identify people's emotions based on video clips from film and TV series to simulate recognition performance in the real world. Researchers from across the world have implemented their latest techniques on the AFEW[2] dataset, which inspires a lot more to come. There are seven categories in the dataset: Angry, Disgust, Fear, Happy, Neutral, Sad, Surprise. In previous years, the winners [3][17][21] have already proposed several efficient methods for multimodal fusion and achieved better results every year. The most common features are non-temporal features, temporal features, and audio features.

Audio features predict emotion status from audio within the video. However, text recognition could be unexpected and confusing since it concerns both context and languages. Other than text, the acoustic features can be beneficial in the multimodal fusion. Here we purpose an acoustic feature extraction model with the state-of-the-art network SoundNet[9].

The static facial expression recognition has also been a promising approach in past challenges[3]. For non-temporal features, the most state-of-the-art techniques such as Inception Net [15] and DenseNet [16] are implemented and constitute an essential part in the final combination.

Features extracted in time-sequence has been proved to be useful in video-based applications. There are two parts: face tracking and temporal features extraction methods. Face tracking can be challenging in the videos taken in the wild. There is a difference between simple face extraction from one single frame and finding a stabilized face window in a video with variant lengths. Efforts have also been made to get better performance in time-sequence from 3D-SIFT [19] to RNN. Developed from RNN, LSTM has shown its potentials in the time-sequence analysis. In the previous EmotiW Audio-Video subtasks for the past few years, LSTM has been widely used in the temporal feature extractions and analysis in Emotion Recognition. On our extended 


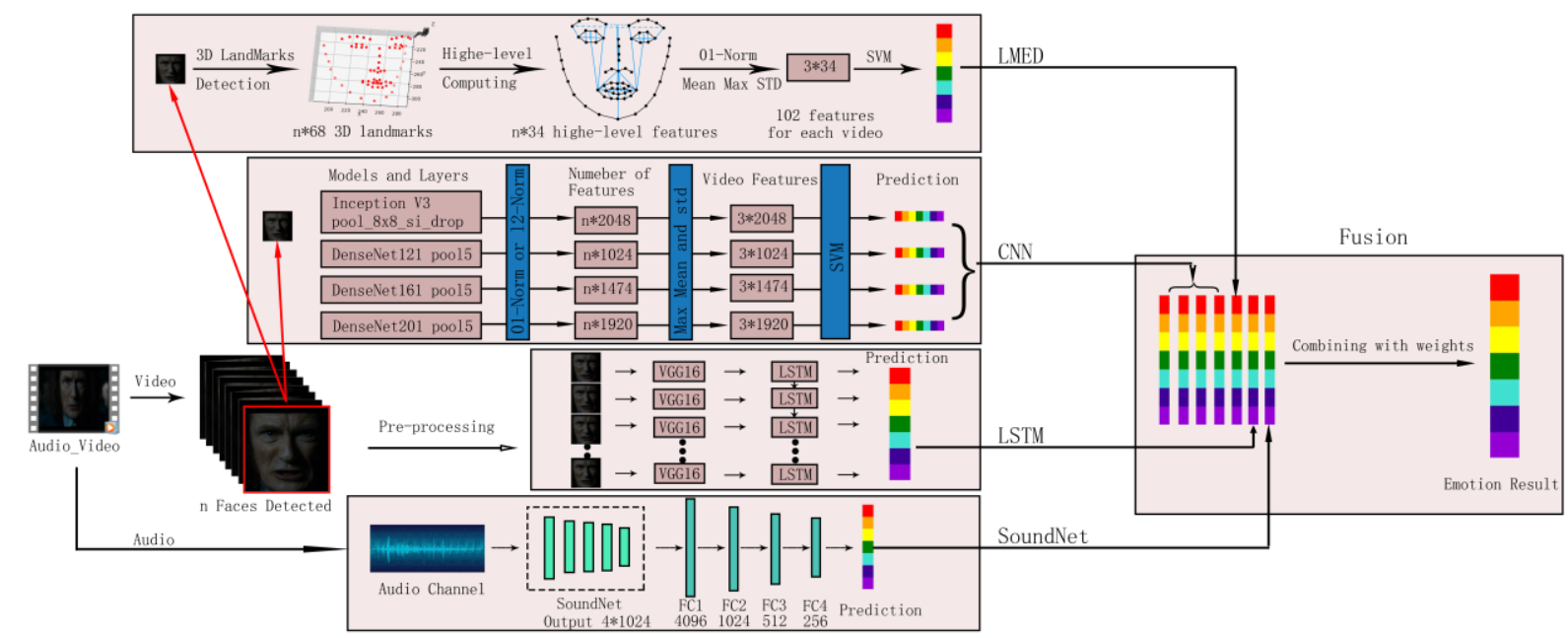

Figure 1: Overview of our multimodal system, there are four main parts in our hybrid net. Every method is described in one pink box and the right down corner shows the name of the method. Each rainbow stripe indicates seven emotion possibilities. The $\mathrm{n}$ in LMDB and CNN box is the same with $\mathbf{n}$ faces detected.

Table 1: STED Databases distribution

\begin{tabular}{ccccccccc}
\hline & Ang & Dis & Fea & Hap & Neu & Sad & Sur & Total \\
\hline \hline Number & 523 & 422 & 379 & 651 & 659 & 486 & 415 & 3535 \\
Rate (\%) & 14.79 & 11.94 & 10.72 & 18.42 & 18.64 & 13.75 & 11.74 & \\
\hline
\end{tabular}

Table 2: The accuracy for different nets on RAF validation dataset. The number in brackets shows the feature dimension of each model that we use for SVM training in the next stage.

\begin{tabular}{|c|c|c|c|c|}
\hline & Ince & enseNe & enseN & Dense \\
\hline & $\left(2048^{*} 3\right)$ & $\left(1024^{*} 3\right)$ & $\left(1474^{*} 3\right)$ & $(1920 * 3)$ \\
\hline$\overline{\mathrm{ccu}}$ & 82.79 & 83.86 & 83.27 & 79.75 \\
\hline
\end{tabular}

validation dataset, VGG-LSTM [21] model turns out to be the most stable and outperformed method. It extracts essential expression features using VGG and analyzes the emotion details on time-sequence. LSTM suits the mission of this challenge which also provides competitive results.

Besides, we introduce 3D facial landmarks changing for classification for this challenge. Several Euclidean distances for landmarks are extracted as another basic feature.

Our main contribution is utilizing facial landmark movements for emotion classification. Besides, we proved the effectiveness of SoundNet in Emotion Recognition tasks.

\section{DATASETS}

\subsection{Image Dataset}

We used the RAF[4] and FER2013[20] datasets to pre-train CNN models. Both datasets contain: Angry, Disgust, Fear, Happy, Neutral, Sad and Surprise. There are 28,709 training, 3,589 validation and 3,589 testing images in FER2013. While the RAF consists of 12,271 training and 3,068 testing samples in the basic folder. It also contains 11 other compound emotion categories which we did not use.

\subsection{The STED Dataset}

Besides AFEW, we collected a large dataset for video clips emotion recognition named Situ Emotion Database (STED). We adopted a similar data collection method with AFEW by abandoning the strategy of using Optical Character Recognition (OCR) information through subtitle and visual data collected. A more accurate two-steps method of manual labeling is proposed. In the first step, our specialists watched the movie on Bilibili and Youku then kept the timestamps. All the movies and TV shows in the AFEW dataset were excluded in the STED. After that, our script automatically saved the video clips in 5 seconds intervals before and after the timestamps. Then, our specialists annotated and edited the 10-second video clips to remove other unnecessary sequences based on their experience. Before that, we ensured that our labelers are thoroughly familiar with the sample of the AFEW training set. All the videos we have collected are formatted in $720 \times 576,25 \mathrm{fps}$, and the length is approximately between 1 and 10 seconds. The distribution of STED dataset shows in Table 1.

\section{METHODS}

\subsection{CNN Feature Based}

3.1.1 Pre-Processing. We extract the frame faces using the MTCNN [5] method which allows us to detect more faces than the dlib [6] detector. Then faces are aligned in a fixed position before data augmentation.

3.1.2 Feature Extracting. We fine-tuned four networks to predict single static images. Table 2 shows the accuracy on RAF validation set. Then we extracted features by the fine-tuned models at the last layers from aligned faces as the basic feature. The feature dimension for each video is relevant to the number of detected face and the layer dimension. Therefore, normalization was introduced to turn different feature dimension to the same. Followed by the previous winners, we calculated the features 
extracted by different CNN models from every aligned face in video frames by mean, max and standard deviation, which makes the video feature three times more than that of what the $\mathrm{CNN}$ model extracted. Then we used the root sift [3] and ranging $[0,1]$ normalization method to deal with the original feature. They can be calculated as follows:

$$
\begin{gathered}
F_{i}^{l 2}=\frac{\left|F_{i}^{v}\right|}{\sum_{j=0}^{n-1}\left(F_{j}^{v}\right)^{2}} \\
F_{i}^{01}=\frac{F_{i}^{v}-\min \left(F^{v}\right)}{\max \left(F^{v}\right)-\min \left(F^{v}\right)}
\end{gathered}
$$

Where $F^{v}$ is the original feature extracted from a video, $F_{i}^{v}$ is the index of feature in the feature vector. $\max \left(F^{v}\right)$ and $\min \left(F^{v}\right)$ stand for the maximum and minimum values in the video feature vector. $F_{i}^{l 2}$ and $F_{i}^{01}$ are the features we normalized by 12-norm and 01-norm. These two normalization methods were trained separately in the next stage as shown in Figure 1.

3.1.3 SVM Training. We trained SVM models with different features which include two kinds of normalization methods and four different networks. Linear SVM was implemented to train the models. We evaluated the parameters by 5 -fold crossvalidation. Table 3 shows the results on AFEW validation set for different features based on the SVM models, which are much lower than the results in RAF static image set. This is because the video clip contains more information about the process of expressions.

\subsection{Landmark Euclidean Distance (LMED)}

Face landmarks are critical high-level features on faces, and experiments have been done by Amit[7] to get the essential landmarks to describe the emotion. Motivated by this method, we calculated 34 Euclidean distances as the face feature (Figure 2, Table 4) by detected 3D Landmarks [8]. For each frame in the video, we got 34 features. In each video, we calculated the mean, maximum and the variance for the corresponding index of the feature as the CNN method did, which means the feature for each video became 102 . We normalized the features only by $01-$ norm method. After that, a linear SVM method was implemented as well. This method archived $39.95 \%$ accuracy on AFEW validation set.

\subsection{Temporal Features}

In this method, MTCNN and SDM[11] are implemented for face tracking. Then we trained our model with VGG facial features on AFEW training dataset and new dataset we collected.

3.3.1 Face tracking and alignment. The difference in poses, illuminations, and occlusions is still a challenging task nowadays. In the time-sequence analysis, the stable face tracking is crucial to its model performance. Here we implement MTCNN for face extraction and SDM for face alignment. Since most frames in the video are known including at least one face, we have tested and chosen a lower face detection threshold, so we can collect more faces and at the same time get fewer errors. As for the SDM, we utilized most of the face landmarks to localize the face of the main character (in most cases, the one with the largest face area).
Table 3: Validation results from different methods on AFEW validation dataset. pool_8x8_s1_drop and pool5 show the layers we extract for each net.

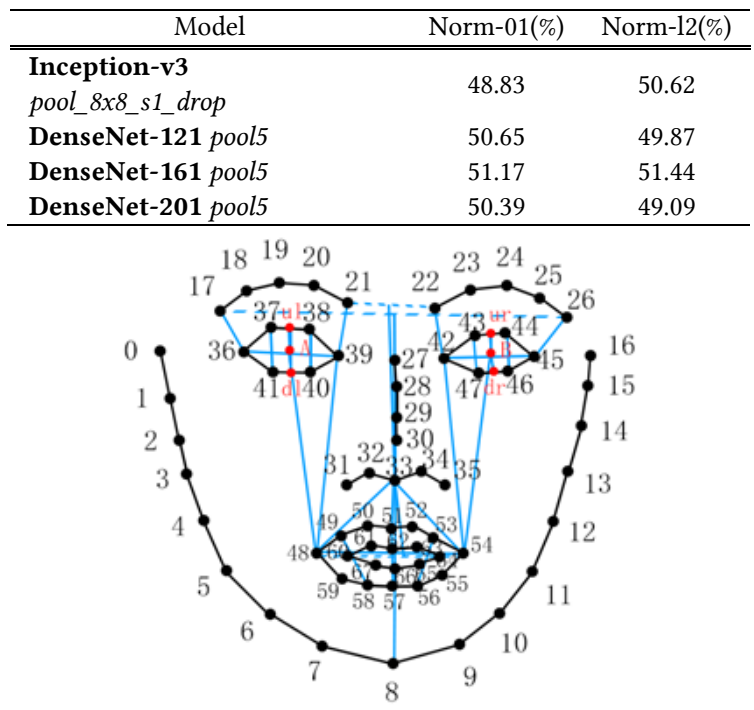

Figure 2: The facial landmarks. The black points can be detected by dlib library, while red marks can be calculated, e.g., point $\mathrm{ul}$ is the middle of 37 and 38 , point $\mathrm{dl}$ is the middle of 40 and 41, point $A$ is the middle of $u l$ and $\mathrm{dl}$. The blue lines are the chosen high-level features who can describe face expression.

Table 4: Facial landmarks description. $\operatorname{dist}(m, n)$ is the distance from point $m$ and point $n$. $\operatorname{mid}(m, n)$ is the middle

\begin{tabular}{|c|c|c|c|c|c|}
\hline $\mathbf{F}$ & D & $\mathbf{F}$ & D & $\mathbf{F}$ & D \\
\hline d0 & $\overline{\operatorname{dist}(17,36)}$ & $\begin{array}{ll}\text { d11 } \\
\end{array}$ & dist(33,48) & d22 & $\frac{\operatorname{dist}(48,54) / 2}{2}$ \\
\hline d1 & $\operatorname{dist}(26,45)$ & d12 & $\operatorname{dist}(33,54)$ & d23 & $\operatorname{dist}(49,58)$ \\
\hline d2 & $\operatorname{dist}(\mathrm{ul}, \mathrm{dl})$ & d13 & $\operatorname{dist}(48,54)$ & d24 & $\operatorname{dist}(53,55)$ \\
\hline d3 & $\operatorname{dist}(37,41)$ & d14 & $\operatorname{dist}(51,57)$ & d25 & $\operatorname{dist}(21,39)$ \\
\hline d4 & $\operatorname{dist}(38,40)$ & d15 & $\operatorname{dist}(33,8)$ & d26 & $\operatorname{dist}(22,42)$ \\
\hline d5 & $\operatorname{dist}(u r, d r)$ & d16 & $\operatorname{dist}(u l, A)$ & d27 & $\operatorname{dist}(36,39) / 2$ \\
\hline d6 & $\operatorname{dist}(43,47)$ & d17 & $\operatorname{dist}(\mathrm{dl}, \mathrm{A})$ & d28 & $\operatorname{dist}(42,45) / 2$ \\
\hline d7 & $\operatorname{dist}(44,46)$ & d18 & $\operatorname{dist}(48, A)$ & d29 & $\mathrm{d} 27+\mathrm{d} 28$ \\
\hline d8 & $\operatorname{dist}(33,51)$ & d19 & $\operatorname{dist}(u r, B)$ & d30 & $\operatorname{dist}(\operatorname{mid}(17,26), 33)$ \\
\hline d9 & $\operatorname{dist}(39,48)$ & d20 & $\operatorname{dist}(\mathrm{dr}, \mathrm{B})$ & d31 & $\operatorname{dist}(\operatorname{mid}(21,22), 33)$ \\
\hline \multirow[t]{2}{*}{ d10 } & $\operatorname{dist}(42,54)$ & d21 & $\operatorname{dist}(54, B)$ & d32 & $\operatorname{dist}(\operatorname{mid}(48,54), 33)$ \\
\hline & & & & d33 & $\operatorname{dist}(62,66)$ \\
\hline
\end{tabular}
point of point $m$ and point $n$.

We also selected a larger window to get more details from areas such as forehead and chin.

3.3.2 VGG Facial Features. We implemented the VGG-16 architecture for facial feature extraction. The reason we choose FER2013 is that FER2013 is well-known for its quality in gray-scale and its adequate numbers of carefully selected faces. With the pretrained VGG-Face Model [12], we fine-tuned our network on FER2013 emotion dataset [13]. The images are sized into 224x224 in grayscale. With data augmentation, we reached $70.96 \%$ accuracy on its validation dataset, which is a state-of-art result. Such performance is an important step before temporal feature extraction. 
Table 5: This table shows the details of our submission. SVM-A means the SVM model for sound. DL-A means the deep model for sound. Based on the $3^{\text {rd }}$ and $6^{\text {th }}$ submission, we added random perturb for fusion weights at the $5^{\text {th }}$ and $7^{\text {th }}$ submission.

\begin{tabular}{|c|c|c|c|c|c|c|c|}
\hline Sub & valid & test & \multicolumn{5}{|c|}{ Fusion detail } \\
\hline - & 38.81 & 40.47 & \multicolumn{5}{|l|}{ Baseline } \\
\hline- & 59.01 & 60.34 & \multicolumn{5}{|c|}{ Best result in Emotiw2017 [17] } \\
\hline 1 & 55.09 & 55.44 & \multicolumn{5}{|c|}{ 4CNNs+LMED - 1} \\
\hline 2 & 56.13 & 53.45 & \multicolumn{5}{|c|}{ 4CNNs+LMED -2 } \\
\hline 3 & 49.61 & 59.11 & \multicolumn{5}{|c|}{ 4CNNs+LMED+Class Weights -1} \\
\hline 4 & - & 59.72 & \multicolumn{5}{|c|}{ 4CNNs+LMED+Class Weights -2} \\
\hline 5 & - & 60.49 & \multicolumn{5}{|c|}{ 4CNNs+LMED+SVM-A+LSTM+class weights } \\
\hline 6 & 50.65 & 61.87 & \multicolumn{5}{|c|}{ 4CNNs LMED+DL-A+LSTM+class weights -1} \\
\hline \multirow[t]{2}{*}{7} & - & 61.71 & \multicolumn{5}{|c|}{ 4CNNs LMED+DL-A+LSTM+class weights -2} \\
\hline & Angry & Disgust & Fear & Happy & Neutral & Sad & Surprise \\
\hline Angry & 71.40 & 0.00 & 3.06 & 3.06 & 18.40 & 4.08 & 0.00 \\
\hline Disgust & 17.50 & 0.00 & 0.00 & 17.50 & 42.50 & 22.50 & 0.00 \\
\hline Fear & 25.70 & 0.00 & 35.70 & 4.29 & 20.00 & 14.30 & 0.00 \\
\hline Happy & 5.56 & 0.00 & 0.69 & 86.10 & 6.25 & 1.39 & 0.00 \\
\hline Neutral & 5.70 & 0.00 & 1.55 & 7.77 & 76.70 & 8.29 & 0.00 \\
\hline Sad & 11.30 & 0.00 & 5.00 & 7.50 & 31.30 & 45.00 & 0.00 \\
\hline Surprise & 3.57 & 0.00 & 25.00 & 14.30 & 28.60 & 25.00 & 3.50 \\
\hline
\end{tabular}

Figure 3: The confusion matrix for our best submission

3.3.3 LSTM. We choose the classic architecture of LSTM which includes a memory cell, an input gate, an output gate and a forget gate. Our implement strategy is just as same as the one in [14]. A training video has been divided into 16-frame clips. The most important step in data augmentation is to divide the video into clips with 8 frames overlap, which has been proved to be effective in both training and testing. Meanwhile, we use mirror and multi-scale methods. The LSTM layer returns the temporal features based on continuing video frames on facial expression. One LSTM layer is applied with 128 embedding outputs. Moreover, the final emotion prediction results on AFEW validation dataset is $46.21 \%$. The one with LSTM-256 layer instead of 128 only achieves $43.07 \%$ in the validation dataset.

\subsection{Audio}

The learning of audio signals plays an important role in improving the performance of our video emotion classification model. In this work, two methods were used, one is the Support Vector Machine (SVM) method which is commonly used, while the other is a deep learning method.

Before applying the commonly used Support Vector Machine (SVM), the first step was to employ OpenSMILE[10] to extract video audio feature with a dimension of 1582. Principal Component Analysis (PCA)[18] was used to reduce the audio feature dimension. Then the RBF SVM was trained on the lower dimensional feature. After grid search, we find the dimension number with the best performance is 513 while the accuracy of the SVM model on the AFEW validation set is $31.07 \%$.

The deep network architecture for audio emotion classification is shown in Figure 3. The entire deep network is the pre-trained
SoundNet [9] model followed by four fully connected layers. The pre-trained SoundNet only contains the first 7 Convolution layers of the original SoundNet model. The input of the deep network architecture is an audio clip with the length of 1 second. The momentum term for Adam optimizer is 0.99 . The model's learning rate is 0.001 . The batch size is 256 . The best accuracy on the validation set achieves $33.16 \%$ in the total 100 training epochs.

\subsection{Fusion}

Chapters 3.1-3.3 show the primary methods. We used each method obtained an emotion prediction for a video. Since emotion is a complex subject, we fused all the different predictions by merely giving weights for the possibilities of each method. The weights were computed by the performance on AFEW validation set and our database set.

\section{RESULTS}

The challenge allowed each team to submit seven times. All the results from our method are displayed in Table 5. For the first two submissions, we only submitted combinations of CNN based models and LMED models. We chose the best two groups of fusion parameters on the AFEW validation set. Guided by feedback, we realized the number for each class is uneven $([98,40$, $70,144,193,80,28])$ which indicates different categories have different levels of significance. In order to deal with this problem, we added two strategies to the follow-up experiments. Previous work shows that adding class weight is a promising method for the unbalanced task [3]. We weighted the score by the square root of the sample numbers $([0.15,0.10,0.13,0.19,0.21$, $0.14,0.08]$ ) which could make our models perform better in categories that are easier to distinguish. Also, we trained our LSTM with our larger dataset which includes more video clips of small sample categories.

The weights for different models came from the experiment on the validation set. The first two submissions contained only CNN models, and class weights were added at the third submission. Audio and LSTM results were added in our last three submissions and our best score achieved at $61.87 \%$ on the test set (Table 5). We also added random perturbs for fusion weights, but we did not get better results.

\section{CONCLUSION}

We present a hybrid multimodal method which includes information consisting of audio, video frame, video sequence and face landmark movement. Compared to the previous winners, our work is an improvement due to the better use of information from the video clips. The lack of certain specimen samples from AFEW dataset indicates that emotion recognition on video clips has not been solved entirely. Our STED dataset, as a supplement, will make this task more manageable to handle. Although we have collected a large dataset ourselves, we did not make full use of it on this challenge because of time limitation. We will do further research and we believe that our dataset will solve the similar task better in the future. 


\section{REFERENCES}

[1] Abhinav Dhall, Amanjot Kaur, Roland Goecke and Tom Gedeon. EmotiW 2018: Audio-Video, Student Engagement and Group-Level Affect Prediction. Proceedings of the 20th. ACM International Conference on Multimodal Interaction 2018 (ACM ICMI 2018). October 16-20, 2018, Boulder, Colorado, USA.

[2] Abhinav Dhall, Roland Goecke, Simon Lucey, and Tom Gedeon. 2012. Collecting Large, Richly Annotated Facial-Expression Databases from Movies. MultiMedia. IEEE 19, 3 (Jul. 2012), 34-41.

[3] Boris Knyazev, Roman Shvetsov, Natalia Efremova and Artem Kuharenko. 2017. Convolutional neural networks pretrained on large face recognition datasets for emotion classification from video. arXiv. 1711, 04598 (Nov. 2017).

[4] Li Shan, Deng Weihong and Du JunPing. 2017. Reliable Crowdsourcing and Deep Locality-Preserving Learning for Expression Recognition in the Wild. 2017 IEEE Conference on Computer Vision and Pattern Recognition (CVPR). IEEE, (Jul. 2007), 2584-2593. DOI: https://doi.org/10.1109/cvpr.2017.277

[5] K. Zhang, Z. Zhang, Z. Li and Y. Qiao. 2016. Joint Face Detection and Alignment Using Multitask Cascaded Convolutional Networks. IEEE Signal Processing Letters. IEEE 23, 10 (Oct, 2016), 1499-1503.

[6] Davis E. King. 2009. Dlib-ml: A Machine Learning Toolkit. fournal of Ma chine Learning Research (Jul. 2009), 1755-1758.

[7] Konar, A. and Chakraborty, A. 2014. Emotion recognition: A pattern analysis approach. John Wiley \& Sons. 138-140.

[8] Bulat, Adrian and Tzimiropoulos, Georgios. 2017. How far are we from solving the 2D \& 3D Face Alignment problem? (and a dataset of 230,000 3D facial landmarks). In Proceedings of the International Conference on Computer Vision. IEEE. DIO: https://doi.org/10.1109/iccv.2017.116

[9] Aytar, Y., Vondrick, C. and Torralba, A., 2016. Soundnet: Learning sound representations from unlabeled video.In Advances in Neural Information Processing Systems (NIPS 2016), 892-900.

[10] Florian Eyben, Martin Wöllmer, and Björn Schuller. 2010. Opensmile: the munich versatile and fast open-source audio feature extractor. In Proceedings of the 18th ACM international conference on Multimedia. ACM, 14591462 .
[11] X. Xiong and F. De la Torre. (2013). Supervised Descent Method and Its Applications to Face Alignment. Computer Vision and Pattern Recognition. IEEE, 532-539.

[12] Parkhi, Omkar M, Andrea Vedaldi and Andrew Zisserman. 2015. Deep face recognition. In British Machine Vision Conference, Vol. 1, No. 3, p. 6.

[13] Ian J Goodfellow, Dumitru Erhan, Pierre Luc Carrier, Aaron Courville, Mehdi Mirza, Ben Hamner, Will Cukierski, Yichuan Tang, David Thaler, DongHyun Lee, and others. 2013. Challenges in representation learning: A report on three machine learning contests. In International Conference on Neural Information Processing. Springer, 117-124.

[14] Donahue, L. A. Hendricks, S. Guadarrama, M. Rohrbach, S. Venugopalan, T. Darrell, and K. Saenko. 2015. Longterm recurrent convolutional networks for visual recognition and description. In Proceedings of the IEEE Conference on Computer Vision and Pattern Recognition. IEEE. 2625-2634.

[15] C. Szegedy, V. Vanhoucke, S. Ioffe, J. Shlens, and Z. Wojna. 2016. Rethinking the inception architecture for computer vision. In Proceedings of the IEEE conference on computer vision and pattern recognition. 2818-2826.

[16] G. Huang, Z. Liu, L. van der Maaten, and K. O. Weinberger. 2017. Densely connected convolutional networks. In Proceedings of the IEEE conference on computer vision and pattern recognition(CVPR). Vol. 1, No. 2, p. 3.

[17] P. Hu, D. Cai, S. Wang, A. Yao, and Y. Chen. 2017. Learning supervised scoring ensemble for emotion recognition in the wild. In Proceedings of the 19th ACM International Conference on Multimodal Interaction. ACM. 553-560.

[18] Wold, Svante, Kim Esbensen, and Paul Geladi. 1987. Principal component analysis. Chemometrics and intelligent laboratory systems. Elsevier 2, 1-3, (Aug. 1987), 37-52.

[19] Scovanner, Paul, Saad Ali, and Mubarak Shah. 2007. A 3-dimensional sift descriptor and its application to action recognition. Proceedings of the 15th ACM international conference on Multimedia. ACM. 357-360.

[20] Ian J Goodfellow, Dumitru Erhan, Pierre Luc Carrier, Aaron Courville, Mehdi Mirza, Ben Hamner, Will Cukierski, and others. 2013. Challenges in representation learning: A report on three machine learning contests. In International Conference on Neural Information Processing. Springer, 117-124.

[21] Fan Y, Lu X, Li D, et al. 2016. Video-based emotion recognition using CNN$\mathrm{RNN}$ and C3D hybrid networks. Proceedings of the 18th ACM International Conference on Multimodal Interaction. ACM. 445-450. 